Journal of Science and Technology Research

Journal homepage: www.nipesjournals.org.ng

\title{
Sedimentation Profile of Strontium Phosphate Precipitate in Aqueous Medium
} Iboroma, Daopuye S. ${ }^{* 1}$; Cookey, Grace A. ${ }^{2}$; Obunwo, Charles C. $^{2}$; Amadi, Kelechi G. ${ }^{2}$

${ }^{1}$ Rivers State Ministry of Environment \& Natural Resources, Port Harcourt, Nigeria

${ }^{2}$ Department of Chemistry, Rivers State University, Port Harcourt, Nigeria

*Corresponding Author Email: daopuye.iboroma1@ust.edu.ng

Phone: +2347060837067

\section{Article Info}

Received 06 June 2020

Revised 22 June 2020

Accepted 23 June 2020

Available online 31 August 2020

Keywords: Precipitate sedimentation, sedimentation profile, strontium phosphate, volume combination, strontium ions, phosphate ions

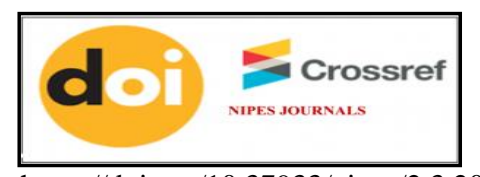

https://doi.org/10.37933/nipes/2.3.2020.12

https://nipesjournals.org.ng (C) 2020 NIPES Pub. All rights reserved

\begin{abstract}
Batch-wise precipitation and sedimentation tests were conducted and diagrams (profiles) describing strontium phosphate $(\mathrm{SrP})$ precipitate sedimentation in aqueous medium were drawn with respect to strontium ions to phosphate ions volume combination. Profiles corresponding to 40:60 and 50:50 strontium ions to phosphate ions volume combinations produced slow SrP precipitate sedimentation comprising of three regions (initial, hindered and compression). On the other hand, fast $\mathrm{SrP}$ precipitate sedimentation consisting of only two regions (hindered and compression) was produced by the 20:80 and 80:20 strontium ions to phosphate ions volume combinations. The observations have been discussed as due mainly to nature of precipitate formed as well as the interactions of the particle with the aqueous medium.
\end{abstract}

\section{Introduction}

Sedimentation play important role in modeling two-phase flow operations and are used to predict the performance of continuous settling tanks [1,2]. Also, sedimentation provides data useful for describing physical stability behaviours of solid phase particles (including bioactive materials) in suspension media [3 - 5].

Models derived from charts that describe sedimentation in discrete, hindered and compression modes abound. Such are one-dimensional and assume particle concentration gradient in the vertical direction only. Simplified form of a model, for example settling profile graph, consider time and height as the only independent variables. Knowledge of such patterns would be useful for predictive sedimentation modeling.

The phenomenon of sedimentation (or settling) is probably the simplest way to separate precipitated or flocculated suspension into concentrated solid and clear supernatant. Conventional sedimentation study involves settling of slurries or suspension introduced into clarification or sedimentation tanks. Contrary to this is precipitate sedimentation involving settling of solid-phase particles generated in reaction vessels, as exchange of ions take place among reacting components. The phenomenon accounts for the deposition of solid-phase particles in industrial water systems as well as in chemical pre-treatment processes such as in sewage treatment plants [6]. Precipitation of insoluble 
compounds and subsequent sedimentation of the insoluble materials remain a cheap and reliable alternative for removal of dissolved ions, for example the hardness ions, from aqueous solution. The combined approach of these two distinct but related phenomena was the experimental basis of a research study titled factors influencing sedimentation characteristics and profiles of alkaline-earth metal phosphates in aqueous solution, embarked upon by Iboroma [7]. Iboroma [7] noted that volume (mixing) ratio of metal and phosphate ions, stirring conditions and temperature of reaction medium influenced sedimentation of alkaline-earth metal phosphate precipitates. A microcontrollerbased precipitate sedimentation measurement device (presed meter) was also designed and used for the research. Details of this study are still being presented. In this paper, sedimentation profiles of strontium phosphate (SrP), described from settling data of SrP precipitate that formed from solutions mixed in various strontium ions to phosphate ions volume combinations, are discussed.

\section{Methodology}

Batch-wise precipitation and sedimentation tests, similar to that by Obunwo et al. (8) were conducted, by mixing equi-molar solutions $(0.1 \mathrm{M})$ of $\mathrm{SrCl}_{2}$ and $\mathrm{Na}_{3} \mathrm{PO}_{4}$ in various volume combinations $(20 \mathrm{ml}: 80 \mathrm{ml}, 40 \mathrm{ml}: 60 \mathrm{ml}, 50 \mathrm{ml}: 50 \mathrm{ml}, 80 \mathrm{ml}: 20 \mathrm{ml})$. Strontium phosphate (SrP) precipitate (as the solid-phase particles) was formed by the ionic reaction of strontium ions and phosphate ions illustrated in Equation (1).

$$
3 \mathrm{Sr}^{2+}{ }_{(\text {aq. })}+2\left(\mathrm{PO}_{4}\right)^{3-}{ }_{(\text {aq. })} \rightarrow \mathrm{Sr}_{3}\left(\mathrm{PO}_{4}\right)_{2(\mathrm{~s})}
$$

To control ambient temperature fluctuations, a microcontroller-based precipitate sedimentation measurement device (Presed Meter) was used to measure sedimentation. Presed meter is an improvement of the precipitate sedimentation measurement apparatus employed in earlier studies $[3,9]$. It consisted of cylindrical tube measuring $25.00 \mathrm{~cm}$ in height and having an inside diameter of approximately $2.80 \mathrm{~cm}$, temperature controlled heating mantle or hot plate, transparent $200 \mathrm{~cm}^{3}$ capacity cylinder (as water-bath), magnetic stirrer, two ultrasonic double-p sensors, stepper motor and microcontroller. The transparent cylindrical tube was the main apparatus in which precipitate sedimentation measurement was done. The heating mantle (Pec Medical Magnetic Stirrer Hotplate - Model Number: 85-2)) provided regulated heat to the sample (in the tube) through the water in the water-bath. To ensure uniform distribution of heat in the water-bath, the magnetic stirrer was positioned to stir the water in the bath without disturbing the sample and the process. The two ultrasonic double-p sensors, transmitter and receiver, suspended by a treaded rod and driven by the stepper motor, faced each other and the cylindrical tube between them. Signal interruption occurs when precipitate was formed in the cylindrical tube. As the signal is interrupted, the microcontroller activates the stepper motor to rotate, thereby driving the sensors in response to the level of the precipitate. The microcontroller interprets the movement of the sensors as well as the corresponding time through LCD displays. Figure 1 is a simplified schematic description of the Presed Meter showing the relationship between major components and their interactions with the sample (precipitate). At a desired temperature setting of the heating mantle (hot plate), the magnetic stirrer distributed heat to the cylindrical tube while the sensors and microcontroller captured and recorded movements made by the precipitate in the tube. The cylindrical tubes (precipitate container and water-bath) being transparent also enabled observations such as colour, nature and volume content of precipitate formed to be made visually. 
For each combination, precipitate formed was stirred for 30 seconds and displacement $(\mathrm{H})$ of the suspension interface with the corresponding time (T) was read from the LCD display of the microcontroller. Sedimentation test lasted for 18 minutes after stirring. Tests were performed at fixed temperature setting $\left(30^{\circ} \mathrm{C}\right)$ of the device. Sedimentation test results were recorded and graphs of $\mathrm{H}$ against $\mathrm{T}$ for 20:80 and 80:20 and 40:60 and 50:50 strontium ions to phosphate ions volume combinations were plotted.

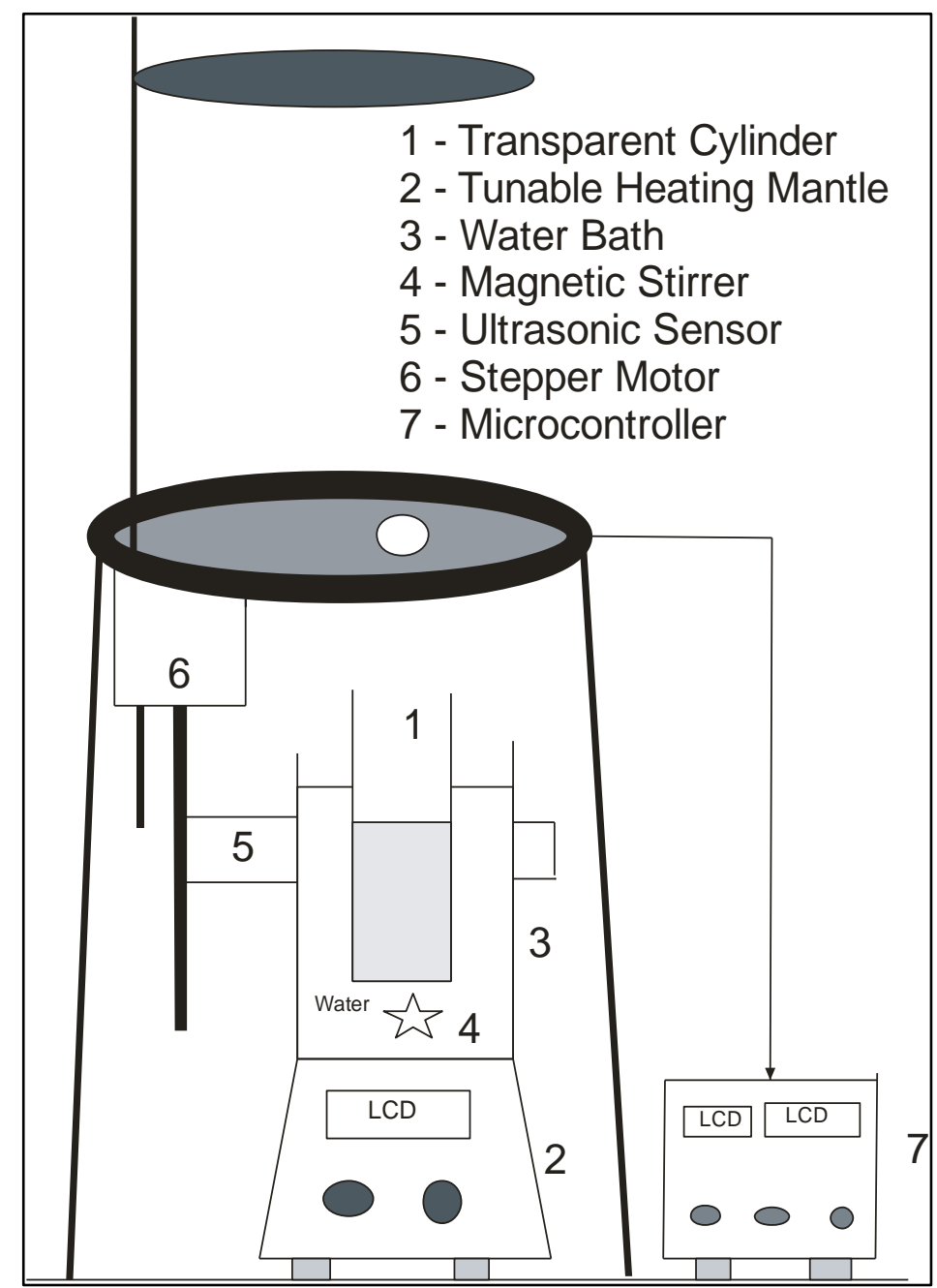

Figure 1: Simplified Schematic of the Precipitate Sedimentation Measurement Device

\section{Results and Discussion}

Results of strontium phosphate ( $\mathrm{SrP}$ ) precipitate sedimentation tests in various strontium ions to phosphate ions volume combinations are given in Figure 2. The displacement per time profiles of SrP precipitate at 20:80 and 80:20 volume combinations are illustrated in Figure 2A. Similarly, Figure 2B showed displacement per time profiles of SrP precipitate at 40:60 and 50:50 volume combinations. Profiles corresponding to 20:80 and 80:20 volume combinations (Figure 2A) produced fast $\mathrm{SrP}$ precipitate sedimentation. Compression apparently started 5 minutes into the settling process. For profiles corresponding to 40:60 and 50:50 volume combinations (Figure 2B), 
compression started from the 14th minute, indicating a relatively slower sedimentation. The most probable explanation for the observation is the interactions of the particles with the aqueous medium (that is, the mother solution). The profiles corresponding to 20:80 and 80:20 volume combinations producing faster sedimentation rate than those corresponding to 40:60 and 50:50, suggest that stronger precipitate-medium interactions occurred when neither strontium ions nor phosphate ions were in excess in the mother solution. Besides, two sedimentation profile patterns can be deduced from test results. The normal profile of interface height or suspension zone plotted against time, containing two regions (Figure 2A) and an alternative profile containing three regions (Figure 2B). The normal and alternative sedimentation profiles are graphical demonstrations of precipitate residence times in suspension. As may be seen, the normal pattern (Figure 2A) described the linear (hindered) and compression regions while the alternative pattern (Figure 2B) described an additional region which can be referred to as initial region. Start-up processes account for the emergence of the initial region and it may be responsible for the long residence times observed for SrP precipitate when solutions of strontium and phosphate ions were mixed at the 40:60 and 50:50 volume combinations. The linear (hindered) and compression zones are commonly encountered and are due to inter-particle hindrance as particles agglomerate together at the bottom of the reaction vessel. The results suggest that SrP precipitate formed at 40:50 and 50:50 volume combinations are colloidal in nature while those of 20:80 and 80:20 volume ratios are rather coarse in nature. The colloidal nature of the precipitate particles formed at 40:50 and 50:50 volume combinations presumably conferred buoyancy on the precipitate particles. Colloidal particles have large-surface-to-mass ratio and an ionic atmosphere known as 'electrical double layer' forms around each colloidal particle [10]. Furthermore, results in Figure 2B suggests that the colloidal size precipitate might have grown to more crystalline (coarse) one in the process of time, in agreement with theory of particle dissolution and re-precipitation [11], and thus shorter residence time immediately after the start-up period.

In precipitate sedimentation, soon after mixing and stirring are undertaken, an initial period of stability of the suspension is usually observed before the on-set of sedimentation. During this period, Brownian forces keep the particles in suspension, especially when the particles are too small. Sedimentation starts when gravitational force on the particle is greater than the Brownian forces. The explanation provided is that during this initial or start-up period, precipitate particles under the influence of Brownian motion, resulting from the agitation (stirring), adjust to a steady state, either by particle growth (deposition) or by flocculation $[12,13]$. Structural transformations such as from a less rigid (amorphous) structure to a more rigid (crystalline or coarse) form may also occur with a corresponding increase in sedimentation rate. Also, chemical interactions of precipitate with the medium, such as those that lead to dissolution of precipitate or formation of entirely new chemical species, may act to alter the trend. In batch precipitation such as was done in this work, concentrations of reactant solutions before mixing as well as their volume (mixing) ratios directly affect the amount and nature of the precipitate particles formed [7]. These properties also affect the interactions of the particles with the aqueous medium and thus physical stability [8]. 
Iboroma, Daopuye $S$. et al. / NIPES Journal of Science and Technology Research 2(3) 2020 pp. 113-118

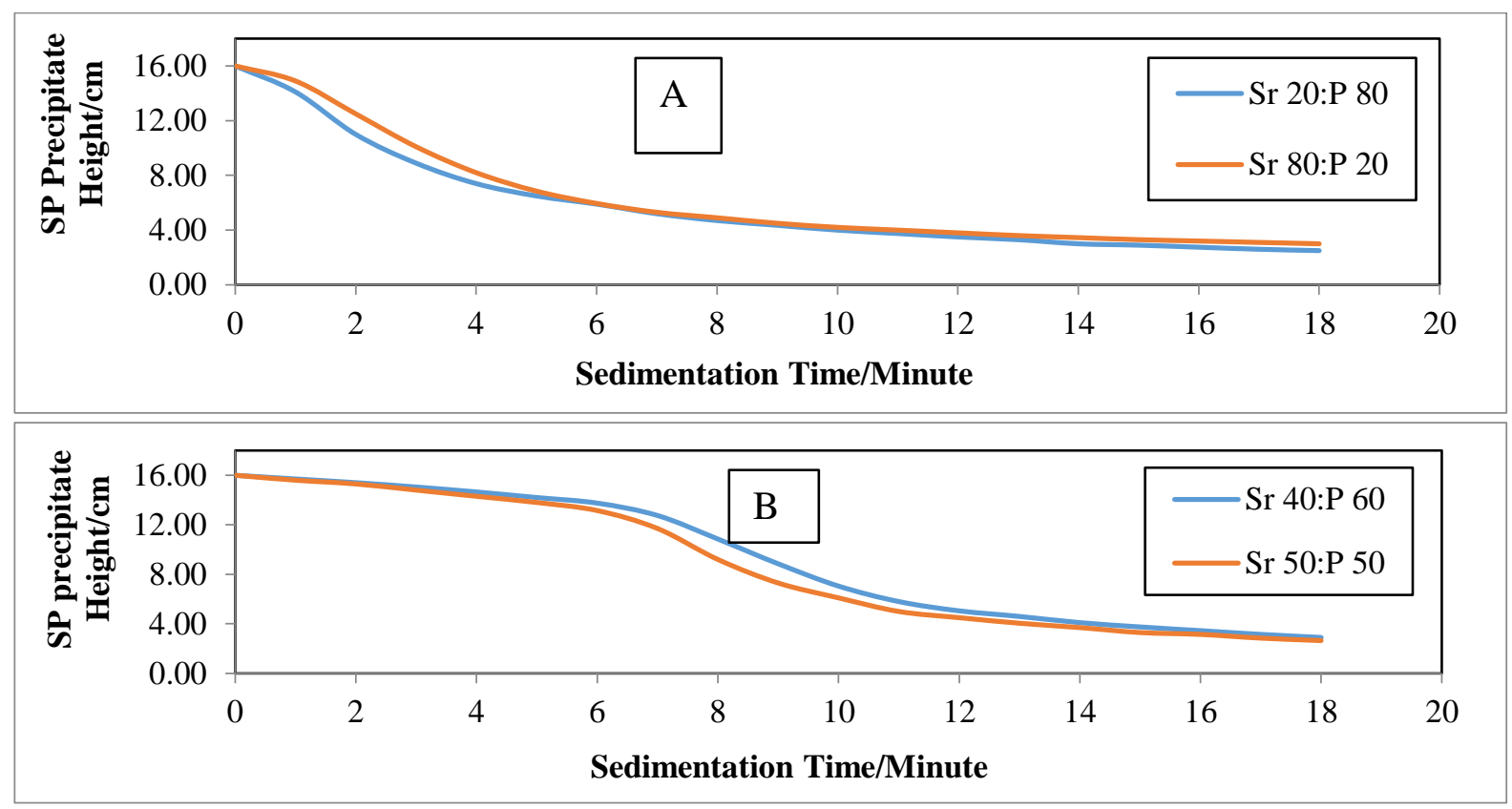

Figure 2: Normal (A) and Alternative (B) Profile Descriptions of SrP Precipitate

\section{Conclusion}

Sedimentation pattern of strontium phosphate ( $\mathrm{SrP})$ in aqueous medium has been described with respect to volume combinations of strontium ions and phosphate ions that formed the precipitate. Fast sedimentation profile, having only two regions, resulted when either the phosphate ions or the strontium ions were in excess in reaction mixture. On the other hand, when neither strontium ions nor phosphate ions were in excess in reaction mixture, slow sedimentation profile, consisting of three regions, resulted. Nature of precipitate formed as well as the interactions of the particle with the aqueous medium has been discussed as likely reason for the observations.

\section{Acknowledgement}

We gratefully acknowledged the inventors of the Presed Meter that was used in making sedimentation measurement. Presed Meter is a patented design of a device that was set up using various detachable apparatuses and appliances. Most of the authors of this research publication contributed in the designing of the device. The inventors have recommended further innovation on the design such as full automation and slimming of its rugged structure into a compact form. However, contributors should seek permission from the original designers.

\section{References}

[1] Holdich, R. G. \& Butt, G. (1997). Solid/liquid separation by sedimentation. Journal of Process Mechanical Engineering, 211 (4), 43 - 45.

[2] Nilufer, K. (2006). A study of channelling behaviour in batch sedimentation. Melbourne,Victoria, Australia (unpublished PhD disertaion). RMIT Univesity.

[3] Iboroma, S. D.; Cookey, G. A. \& Obunwo, C. C. (2018). Effect of sodium carboxymethylcellulose biopolymer on sedimentation properties of trimagnesium, tricalcium and tristrontium phosphates precipitates. Journal Applied Science Environmental Management, 22 (10), 1591 - 1594. 
[4] Iboroma, S. D.; Cookey, G. A. \& Obunwo, C. C. (2019a). Sedimentation study of tribasic magnesium phosphate precipitate in aqueous medium. International Journal of Applied Science and Research, 2 (2): 1 5 .

[5] Chabni, M.; Bougherra, H.; Lounici, H.; Ahmed-Zaid, T.; Canselier, J. \& Bertrand, J. (2011). Evaluation of physical stability of zinc oxide suspensions containing sodium poly-(acrylate) and sodium dodecyl sulfate. Journal of Dispersion Science and Technology, 32 (12), 1786 - 1798.

[6] Iboroma, D. S.; Cookey, G. A.; Obunwo, C. C. \& Amadi, K. G. (2020). Evaluation of the Effects of Temperature and Ion Concentration Variables on Sedimentation of Magnesium and Barium Phosphates in Aqueous Media. International Journal of Scientific and Research Publications, 10, (2), 565 - 571.

[7] Iboroma, S. D. (2019). Factors influencing sedimentation characteristics and profiles of alkaline-earth metal phosphates in aqueous solution. Port Harcourt, Rivers State, Nigeria (unpublished PhD Thesis). Rivers State University.

[8] Obunwo, C. C; Iboroma, S. D. \& Bagshaw, A. P. (2017). Effects of physical variables on settling velocities of calcium and strontium phosphates in mother solution. Journal. Applied Science \& Environmental Management, 21 (2), 307 - 311.

[9] Iboroma, S. D.; Obunwo, C. C. \& Cookey, G. A. (2019b). Sedimentation behaviour of barium phosphate precipitate in aqueous solution. International Journal of Applied Science and Research, 2 (2), 1 - 6.

[10] Obunwo, C. C. \& Iboroma, D. S. (2015). Determination of effects of some parameters on the sedimentation rates of strontium and barium carbonates in aqeous medium. IOSR Journal of Applied Chemistry, 8 (2), 17 21.

[11] Skoog, G. A.; West, D. M. \& Holler, F. J. (1995). Fundamentals of Analytical Chemistry (7 ${ }^{\text {th }}$ ed.). Philadelphia, PA: Saunders College.

[12] Punnamaraju, S. R. (2012). The evaluation of the sedimentation behaviour of magnesium hydroxide in the never dried state. Toledo, Ohio, USA (unpublished MSc Dissertation). University of Toledo.

[13] Jain, R. (2014). The evaluation of the hindered settling behaviour of the ground calcium carbonate suspension. Toledo, Ohio, USA (unpublished MSc Dissertation). University of Toledo. 